\title{
A hybrid non-dominated sorting genetic algorithm for a multi-objective demand-side management problem in a smart building
}

\author{
Zineb Garroussi ${ }^{1}$, Rachid Ellaia ${ }^{2}$, El-Ghazali Talbi ${ }^{3}$, Jean-YvesLucas ${ }^{4}$ \\ ${ }^{1,2}$ LERMA Laboratory, Engineering for Smart and Sustainable Systems Research Center (E3S), \\ Mohammadia School of Engineers, Mohammed V University of Rabat, Morocco \\ ${ }^{3}$ Big Optimization and Ultra-Scale computing team (BONUS), Inria Lille - Nord Europe Research Centre, France \\ ${ }^{4}$ Electricité de France S.A, EDF R\&D, avenue du Général de Gaulle, France
}

\begin{abstract}
Article Info
Article history:

Received Jun 17, 2019

Revised Sep 24, 2019

Accepted Oct 1, 2019

\section{Keywords:}

Demand-side management

Electrical loads

Energy source

Energy storage

Evolutionary algorithm

Matheuristic

Multi-objective optimization

Thermal loads

ABSTRACT

One of the most significant challenges facing optimization models for the demand-side management (DSM) is obtaining feasible solutions in a shorter time. In this paper, the DSM is formulated in a smart building as a linear constrained multi-objective optimization model to schedule both electrical and thermal loads over one day. Two objectives are considered, energy cost and discomfort caused by allowing flexibility of loads within an acceptable comfort range. To solve this problem, an integrative matheuristic is proposed by combining a multi-objective evolutionary algorithm as a master level with an exact solver as a slave level. To cope with the non-triviality of feasible solutions representation and NP-hardness of our optimization model, in this approach discrete decision variables are encoded as partial chromosomes and the continuous decision variables are determined optimally by an exact solver. This matheuristic is relevant for dealing with the constraints of our optimization model. To validate the performance of our approach, a number of simulations are performed and compared with the goal programming under various scenarios of cold and hot weather conditions. It turns out that our approach outperforms the goal programming with respect to some comparison metrics including the hypervolume difference, epsilon indicator, number of the Pareto solutions found, and computational time metrics.
\end{abstract}

Copyright $\odot 2020$ Institute of Advanced Engineering and Science. All rights reserved.

\section{Corresponding Author:}

Zineb Garroussi,

LERMA Laboratory, Engineering for Smart and Sustainable Systems Research Center (E3S),

Mohammadia School of Engineers, Mohammed V University of Rabat,

BP. 765, Ibn Sina av, Rabat, Morocco.

Email: zineb.garroussi@gmail.com

\section{INTRODUCTION}

With the growth of innovative electronics systems, the building technologies have witnessed tremendous developments, giving rise to a smart building concept [1] . A smart building is a fully connected building, equipped with information and communication technologies that can respond continuously to the changing inhabitant requirements and environmental conditions, to provide safety and convenience of living [2]. Demand-side management (DSM) in a smart building can be defined as a set of programs that induce energy consumers to use energy in a rational way. Indeed, the DSM provides a multitude of benefits for the futuristic smart building by reducing energy costs and promoting the incorporation of clean renewable and low carbon energy technologies [3]. Among the reliable DSM services, demand response encourages consumers to voluntary reducing or shifting energy usage during peak periods in response to time-based rates 
or other forms of financial incentives. For instance, in a smart building, the smart meter receives external price signal such as time of use pricing (ToU) which vary depending on three periods, off-peak, mid-peak and on-peak price periods, and then all connected energy devices are scheduled to give a better economic planning, but affecting consumer preferences, so that it might be operated at undesired periods. However, in such circumstances, considering a trade-off between the economic targets of the DSM and a convenient lifestyle is substantial. The automatic implementation of the DSM scheduling model in a smart building (B-DSM) is performed by using a home/building energy management system (HEMS/BEMS) and is becoming a challenging with respect to diversity of building appliances, targets and constraints to be optimized.

Different B-DSM optimization models for the HEMS or BEMS have been established in the literature. For instance in [4], an appliance-scheduling is proposed taking into account a photovoltaic panel and a hybrid electric vehicle (PHEV). A dynamic multi-swarm with learning strategy is applied to solve the proposed model with the objective of reducing the weighted sum of the electricity payments, consumer's dissatisfaction and carbon dioxide emissions. In [5] the B-DSM with household appliances and a battery is proposed. The authors minimize the electricity cost and the discomfort level. The discomfort is modeled through the disparity between the baseline and the optimal schedule. The mixed integer non-linear optimization model is built and solved with AIMMS software. Authors in [6] proposed a framework for the HEMS with a battery, PV output and electric vehicle. They developed an improved particle swarm optimization with a heuristic approach to schedule appliances and calculate power distribution under electricity time varying prices, user preference constraint. The objective is the weighted sum of the electricity cost and the discomfort. In [7] a mixed integer non-linear optimization model is built for the DSM in a smart home considering electrical and thermal appliances, a battery and a cogeneration appliance. In [8], authors formulated the B-DSM as a multi-objective model between the electricity cost and the discomfort. The discomfort takes two aspects: Time slot preferences, and risk of interruption of energy supply. They applied a non-dominated sorting genetic algorithm (NSGAII) to find the compromise between the electricity cost and the discomfort.

The majority of practical HEMS/BEMS optimization models are designed as mixed-integer linear programming problems (MILPs) with more than one objective. Getting feasible solutions in a shorter time is one of the most important challenges that face optimization models for the DSM in smart buildings. MILPs are NP-hard problems involving discrete and continuous decision variables. Exact solvers such CPLEX and GAMS which implement branch-and-bound or cutting planes algorithms are suitable for small sizedproblems for finding optimal solutions within a reasonable computation time. However, when the number of integer and/or binary variables grows in medium and large MILP problems, these algorithms are extremely time-consuming and have exponential computing complexity [9]. To use an exact solver in less time, some existing works proposed an integer-free optimization model for energy management in order to reduce the computation time [10]. However, this work ignores the scheduling of on/off status of appliances.

Classical techniques for dealing with multi-objective optimization problems convert the multiobjective optimization problem (MOOP) to a single-objective optimization problem (SOOP). The epsilonconstraint method involves optimizing one objective and use all other objectives as constraints [9, 11], the WSA involves optimizing a weighted sum of various objectives [12], the weighted metric methods indicate optimizing an $1_{p}$ metric constructed from all objectives [13], and the goal programming technique involves optimizing a weighted sum of deviations of objectives from user-specified targets [14]. The arised SOOP should be tackled using a single-optimization algorithm. Each solution from the SOOP is specific to the conversion parameters. However, the disadvantages of these conversions are requiring several calls to the single-objective algorithm in order to obtain a set of Pareto optimal solutions, requiring some problem knowledge, such as suitable target values or weights, and having limitations to obtain solutions in the non-convex region of the Pareto optimal set [15].

Multi-objective evolutionary algorithms (MOEA) which imitate the natural selection process are well-adapted for MOOP. Among the most popular MOEA algorithm, NSGAII is a population-based metaheuristic, it aims to enhance population of solutions iteratively by using evolutionary operators (selection, crossover and mutation), and classifying the population into sub-populations based on Pareto dominance order and crowding distance concepts. However, NSGAII doesn't ensure finding an optimal Pareto front, and for some highly-constrained MOOP, the encoding of feasible candidate solutions isn't straightforward and involving the developement of some problem-specific heuristics [16].

In this paper, we propose a B-DSM optimization model in order to coordinate a typical set of appliances during 24 hours for both hot and cold weather conditions. These appliances are classified into three categories: Local energy sources, local energy storages, and loads. Local energy sources include photovoltaic panels (PV) and micro-combined heating cooling and power appliances (mCCHP). Local energy storages contain electrical domestic batteries (B) and thermal energy storages (TES). Loads are 
subdivided into: thermal loads, and electrical loads. Thermal loads (TL) use heat produced by mCCHPs or stored in TES and include storage water heater appliances (SWH) and Heating \& Air Conditioning appliances (HAC). Electrical loads (EL) include time shiftable loads (TSL), non-controllable loads (NCL) and electric chillers when the hot day is considered.

To ovecorme the NP-hardness with the number of discrete (integer and binary) variables and guarantee the validity of solutions in our highly constrained mixed-integer linear scheduling model, we resort to a matheuristic to solve it within a reasonable computational time. In general, matheuristics are algorithms that combine mathematical programming methods and metaheuristics [17]. Our proposed hybrid approach is an integrative combination where the NSGAII works at the higher level and the exact algorithm is a subordinate embedded component of the evolutionary algorithm. More precisely, in our proposed matheuristic called (H-NSGAII) solutions are incompletely encoded, and an exact algorithm which is a black box solver (CPLEX) used as a decoder for determining the missing parts in an optimal fashion. This indirect encoding/decoding strategy is efficient in optimization problems involving many and complex constraints to satisfy, such as scheduling problems where neither metaheuristics nor mathematical programming is able to find good quality solutions within an acceptable computational time. To validate the performance of our approach, a number of simulations are performed and compared with the goal programming (GP) method under various scenarios. It turns out that our approach outperforms the GP method with respect to some comparison metrics including the hypervolume difference, epsilon indicator, number of Pareto solutions found and computational time metrics. In summary, the major contributions of this paper are: (i) solving our B-DSM faster than an exact solver; (ii) Overcoming the challenge of developing a problem-specific heuristics for representation of feasible solutions when implementing a MOEA on our constrained MILP problem; (iii) showing the effectiveness of our matheuristic in obtaining a set of Pareto optimal solutions between the total energy cost and the total discomfort while satisfying all constraints.

The rest of this paper is organized as follows. In section 2, we formulate the mathematical model for the multi-objective B-DSM. We then describe the proposed matheuristic approach in section 3 . In section 4, we provide data, scenarios, simulation results and discussions. Section 5 gives conclusions and future perspectives.

\section{B-DSM OPTIMIZATION MODEL}

In this section, we model the different components involved in the proposed B-DSM. A shown in Figure 1. The mCCHP system is modeled to generate electrical, heating and cooling powers. It is composed of a power generation unit (PGU) to produce electrical power from the natural gas whereas the recovering waste heat from the PGU is employed by the heat recovery system to satisfy the heat demand. The excess heat could be stored in the TES and can be later utilised to supply the needed thermal power. The cooling demand of our B-DSM is whether met by the absorption chiller (AC) or electric chiller (EC) [18]. Based on the day-ahead time of use tariff and gas price, all building components are then scheduled. For each time slot $t \in T$ where $T$ is the time horizon, $P_{P V 2 E L}^{i, t}, P_{P V 2 G}^{i, t}$ and $P_{P V 2 B}^{i, t}$ are the electrical powers supplied from the i-th PV to the electrical loads, the grid, and the battery respectively. $P_{m C C H P 2 E L}^{i, t}, P_{m C C H P 2 G}^{i, t}, P_{m C C H P 2 B}^{i, t}$ are the electrical power injected from the i-th mCCHP to the electrical loads, the grid, and the battery respectively. $P_{G 2 B}^{t}$ and $P_{G 2 E L}^{t}$ are the electrical powers supplied from the grid to the batteries and the electrical loads respectively. $P_{B 2 E L}^{i, t}$ is the electrical power injected from the i-th battery to the electrical loads.

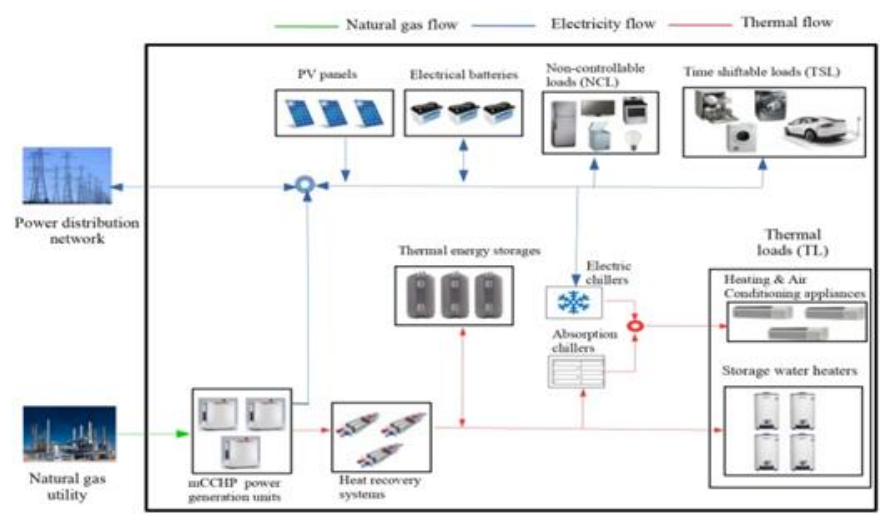

Figure 1. Proposed B-DSM framework 


\section{1. mCCHP model}

The relation between the electrical and thermal power output $\left(P_{\mathrm{mCCHP}}^{i, t}\right.$ and $\left.H_{\mathrm{mCCHP}}^{i, t}\right)$ of the i-th mCCHP is expressed as (1) [19]. $\eta_{i, e}$ and $\eta_{i, t h}$ are the electrical and thermal efficiencies of the i-th mCCHP respectively. $n_{m C C H P}$ is the number of the considered mCCHPs in the building. The natural gas consumption $F_{\text {mCCHP }}^{i, t}$ of the $\mathrm{i}$-th $\mathrm{mCCHP}$ in $\mathrm{m}^{3}$ is given as (2) $\beta$ is the converting factor of $1 \mathrm{kWh}^{2}$ to $\mathrm{m}^{3}$ natural gas. To avoid stressed mCCHPs generations, minimum/maximum Up-Time, and minimum Down-Time constraints are insured with the constraints [20]. Minimum Up-Time constraint (3) defines the minimum time the i-th mCCHP should be started up once it is running. The i-th mCCHP initial and final status should satisfy the constraints (4) and (5). $T_{\mathrm{up}}^{i, \min }$ and $T_{\mathrm{up}_{0}}^{i, \min }$ are the minimum uptime and the initial minimum uptime of the i-th mCCHP respectively. Maximum Up-Time constraint (6) defines the maximum time the i-th mCCHP has to be on once it started up. $T_{\text {up }}^{i, \max }$ is the maximum uptime of the i-th mCCHP. Minimum Down-Time constraint (7) signifies the minimum time for which the i-th mCCHP should be turned off once a shutdown occurs. The initial and final time steps should satisfy the constraints (8) and (9) respectively. $T_{\text {down }}^{i, m i n}$ and $T_{\text {down }_{0}}^{i, m i n}$ are, respectively, the minimum downtime and initial minimum downtime of the i-th mCCHP. The i-th mCCHP electrical and heat power must range between their minimum and maximum capacities, as expressed in the contraints $(10)$ and $(11) . P_{\mathrm{mCCHP}}^{i, \min }\left(H_{\mathrm{mCCHP}}^{i, \min }\right)$ and $P_{\mathrm{mCCHP}}^{i, \max }\left(H_{\mathrm{mCCHP}}^{i, \max }\right)$ are the allowable minimum and maximum electrical (thermal) output of the $\mathrm{i}$-th mCCHP respectively.

The $s_{\text {mCCHP }}^{i, t}$ is a binary variable corresponding to the on/off status of the i-th mCCHP at time slot $t$. The ramping constraints ensure that the $\mathrm{i}$-th $\mathrm{mCCHP}$ production variations between two successive time slots are limited as expressed as in (12) and (13). $r r_{i}$ is the ramp rate of the i-th $\mathrm{mCCHP}(\mathrm{kW} / \mathrm{h})$.

$$
\begin{aligned}
& P_{m C C H P}^{i, t}=\frac{\eta_{i, e}}{\eta_{i, t h}} \cdot H_{m C C H P}^{i, t} \forall i \in\left\{1, \ldots, n_{m C C H P}\right\}, \forall t \in\{1, \ldots, T\} \\
& F_{m C C H P}^{i, t}=\frac{P_{m C C H P}^{i, t}}{\eta_{i, e}} \cdot \beta \quad \forall i \in\left\{1, \ldots, n_{m C C H P}\right\}, \forall t \in\{1, \ldots, T\} \\
& \sum_{n=t}^{t+T_{u p}^{i, m i n}-1} s_{m C C H P}^{i, n} \geq T_{u p}^{i, m i n} \cdot\left(s_{m C C H P}^{i, t}-s_{m C C H P}^{i, t-1}\right) \quad \forall t=\left\{T_{u p_{0}}^{i, m i n}+1, \ldots, T-T_{u p}^{i, m i n}+1\right\} \\
& \sum_{t=1}^{T_{u p_{0}}^{i, \min }}\left(1-s_{m C C H P}^{i, t}\right)=0 \quad \forall i \in\left\{1, \ldots, n_{m C C H P}\right\} \\
& \sum_{n=t}^{T}\left(s_{m C C H P}^{i, n}-\left(s_{m C C H P}^{i, t}-s_{m C C H P}^{i, t-1}\right)\right) \geq 0 \forall i \in\left\{1, \ldots, n_{m C C H P}\right\} \forall t=\left\{T-T_{u p}^{i, m i n}+2, \ldots, T\right\} \\
& \sum_{n=t}^{t+T_{u p}^{i, m a x}} s_{m C C H P}^{i, n} \leq T_{u p}^{i, \max } \forall i \in\left\{1, \ldots, n_{m C C H P}\right\} \forall t=\left\{1, \ldots, T-T_{u p}^{i, \max }\right\} \\
& t+T_{\text {down }}^{i, m i n} \\
& \sum_{n=t}^{d o w}\left(1-s_{m C C H P}^{i, n}\right) \geq T_{\text {down }}^{i, m i n} \cdot\left(s_{m C C H P}^{i, t-1}-s_{m C C H P}^{i, t}\right) \forall t=\left\{T_{\text {down }}^{\min }+1, \ldots, T-T_{\text {down }}^{\min }+1\right\} \\
& \sum_{t=1}^{T_{\text {down }}^{i, m i n}} s_{m C C H P}^{i, t}=0 \\
& \sum_{n=t}^{T}\left(1-s_{m C C H P}^{i, n}-\left(s_{m C C H P}^{i, t-1}-s_{m C C H P}^{i, t}\right)\right) \geq 0 \forall t=\left\{T-T_{d o w n}^{i, m i n}+2, \ldots, T\right\}
\end{aligned}
$$




$$
\begin{aligned}
& s_{m C C H P}^{i, t} \cdot P_{m C C H P}^{i, m i n} \leq P_{m C C H P}^{i, t} \leq s_{m C C H P}^{i, t} \cdot P_{m C C H P}^{i, m a x} \forall i \in\left\{1, \ldots, n_{m C C H P}\right\}, \forall t \in\{1, \ldots, T\} \\
& s_{m C C H P}^{i, t} \cdot H_{m C C H P}^{i, m i n} \leq H_{m C C H P}^{i, t} \leq s_{m C C H P}^{i, t} \cdot H_{m C C H P}^{i, m a x} \forall i \in\left\{1, \ldots, n_{m C C H P}\right\}, \forall t \in\{1, \ldots, T\} \\
& \left|P_{m C C H P}^{i, t}-P_{m C C H P}^{i, t-1}\right| \leq r r_{i} \forall i \in\left\{1, \ldots, n_{m C C H P}\right\}, \forall t \in\{2, \ldots, T\} \\
& \left|H_{m C C H P}^{i, t}-H_{m C C H P}^{i, t-1}\right| \leq \frac{\eta_{i, t h}}{\eta_{i, e}} \cdot r r_{i} \forall i \in\left\{1, \ldots, n_{m C C H P}\right\}, \forall t \in\{2, \ldots, T\}
\end{aligned}
$$

\subsection{TES model}

The heat power injected $\mathbf{H}_{\mathbf{i n}}^{\mathbf{i}, \mathbf{t}}$ and drawn $\mathbf{H}_{\mathbf{d r}}^{\mathbf{i}, \mathbf{t}}$ from the i-th TES are bounded as in (14) and (15) respectively. $\mathbf{s}_{\mathbf{i n}}^{\mathbf{i}, \mathbf{t}}$ and $\mathbf{s}_{\mathbf{d r}}^{\mathbf{i}, \mathbf{t}}$ are binary variables which mean the injecting and drawing status at time slot $\mathrm{t}$. nTES is the number of the considered TES in the building. $\eta$ i,in and $\eta$ i,dr are the injecting and drawing heat efficiencies. $\mathbf{H}_{\mathbf{i n}}^{\mathbf{i}, \mathbf{m a x}}$ and $\mathbf{H}_{\mathbf{d r}}^{\mathbf{i}, \boldsymbol{m a x}}$ are the maximal heat injected and drawn respectively. The constraint (16) determines the power flow of the $\mathrm{i}$-th TES in any given time slot $\mathrm{t}$. The $\mathrm{i}$-th TES energy content $\mathbf{Q}_{\text {TES }}^{\mathbf{i}, \mathbf{t}}$ is modeled by (17). The i-th TES energy content is limited as (18). $\mathbf{Q}_{\text {TES }}^{\mathbf{i}, \mathbf{m i n}}$, and $\mathbf{Q}_{\mathrm{TES}}^{\mathbf{i}, \mathbf{m a x}}$ are the minimum and the maximum energy content limits of the $\mathrm{i}$-th TES. In order that the $\mathrm{i}$-th TES can fulfil the requirement for the next day, the initial and final energy content, denoted $\mathbf{Q}_{\text {TES }}^{\mathbf{i} \mathbf{1}}$ and $\mathbf{Q}_{\text {TES }}^{\mathbf{i}, \mathbf{T}}$ are fixed on the same predetermined value.

$$
\begin{aligned}
& \mathbf{0} \leq \boldsymbol{H}_{\boldsymbol{i n}}^{\boldsymbol{i}, \boldsymbol{t}} \leq \frac{\mathbf{1}}{\boldsymbol{\eta}_{\boldsymbol{i}, \boldsymbol{i n}}} \cdot \boldsymbol{H}_{\text {in }}^{\boldsymbol{i}, \boldsymbol{m a x}} \cdot \boldsymbol{s}_{\boldsymbol{i n}}^{\boldsymbol{i}, \boldsymbol{t}} \forall \boldsymbol{i} \in\left\{\mathbf{1}, \ldots, \boldsymbol{n}_{\boldsymbol{T} E S}\right\}, \forall \boldsymbol{t} \in\{\mathbf{1}, \ldots, \boldsymbol{T}\} \\
& 0 \leq H_{d r}^{i, t} \leq H_{d r}^{i, m a x} \cdot s_{d r}^{i, t} \cdot \eta_{i, d r} \forall i \in\left\{1, \ldots, n_{T E S}\right\}, \forall t \in\{1, \ldots, T\} \\
& s_{i n}^{i, t}+s_{d r}^{i, t} \leq 1 \forall i \in\left\{1, \ldots, n_{T E S}\right\}, \forall t \in\{1, \ldots, T\} \\
& Q_{T E S}^{i, t+1}=Q_{T E S}^{i, t}+\left(H_{i n}^{i, t} \cdot \eta_{i, i n}-\frac{H_{d r}^{i, t}}{\eta_{i, d r}}\right) \forall i \in\left\{1, \ldots, n_{T E S}\right\}, \forall t \in\{1, \ldots, T\} \\
& Q_{T E S}^{i, m i n} \leq Q_{T E S}^{i, t} \leq Q_{T E S}^{i, \max } \forall i \in\left\{1, \ldots, n_{T E S}\right\}, \forall t \in\{1, \ldots, T\}
\end{aligned}
$$

\subsection{Battery model}

The state of charge $\mathbf{S O C}_{\mathbf{i}, \mathbf{t}}$ of the i-th battery is given by (19). $\mathrm{n}_{\mathrm{B}}$ is the number of batteries. $\mathbf{P}_{\mathbf{c h}}^{\mathbf{i}, \mathbf{t}}$ and $\mathbf{P}_{\mathbf{d c h}}^{\mathbf{i}, \mathbf{t}}$ determine the battery charging and discharging rates of the i-th battery. Ei, $\eta \mathrm{i}$,ch and $\eta \mathrm{i}$,dch are the capacity, charging and discharging efficiencies of the i-th battery. The SOCi,t is bounded as expressed in (20) [21]. $\mathbf{S O C} \mathbf{C}_{\mathbf{i} \text { min }}$ and $\mathbf{S O C} \mathbf{C}_{\mathbf{i} \text { max }}$ are, respectively, the maximal and minimal state of charge of the i-th battery. The $\mathbf{P}_{\mathbf{c h}}^{\mathbf{i}, \mathbf{t}}$ and $\mathbf{P}_{\mathbf{d c h}}^{\mathbf{i}, \mathbf{t}}$ of the i-th battery are limited by the constraints (21), (22). $\mathbf{P}_{\mathbf{c h}}^{\mathbf{i}, \mathbf{m a x}}$ (resp. $\mathbf{P}_{\mathbf{d c h}}^{\mathbf{i}, \mathbf{m a x}}$ ) is the maximal charging (resp. discharging) rate of the i-th battery. $\mathbf{s}_{\mathbf{c h}}^{\mathbf{i}, \mathbf{t}}$ and $\mathbf{s}_{\mathbf{d c h}}^{\mathbf{i}, \mathbf{t}}$ express the charging/discharging status of the $\mathrm{i}$-th battery. $\mathbf{s}_{\mathbf{c h}}^{\mathbf{i}, \mathbf{t}}$ (resp. $\mathbf{s}_{\mathbf{d c h}}^{\mathbf{i}, \mathbf{t}}$ ) is equal to 1 if the i-th battery is charged (resp. discharged) at time slot $t$ and 0 otherwise. The battery usage constraint shows that the i-th battery in a specific time slot $t$ can be in one of three modes: charge, discharge or off as given in (23). The charging can be from all installed mCCHPs, PVs or the main grid (24). The discharging can only to the electrical loads (25).

$$
\begin{aligned}
& S O C^{i, t+1}=S O C^{i, t}+\frac{P_{c h}^{i, t} \cdot \eta_{i, c h}}{E_{i}}-\frac{P_{d c h}^{i, t}}{E_{i} \cdot \eta_{i, d c h}} \forall i \in\left\{1, \ldots, n_{B}\right\}, \forall t \in\{1, \ldots, T\} \\
& S O C^{i, \min } \leq S O C^{i, t} \leq S O C^{i, \max } \forall i \in\left\{1, \ldots, n_{B}\right\}, \forall t \in\{1, \ldots, T\} \\
& \mathbf{0} \leq \boldsymbol{P}_{\boldsymbol{c h}}^{i, \boldsymbol{t}} \leq \boldsymbol{s}_{\boldsymbol{c h}}^{i, \boldsymbol{t}} \cdot \boldsymbol{P}_{\boldsymbol{c h}}^{i, \boldsymbol{m a x}} \forall \boldsymbol{i} \in\left\{\mathbf{1}, \ldots, \boldsymbol{n}_{\boldsymbol{B}}\right\}, \forall \boldsymbol{t} \in\{\mathbf{1}, \ldots, \boldsymbol{T}\} \\
& 0 \leq P_{d c h}^{i, t} \leq s_{d c h}^{i, t} \cdot P_{d c h}^{i, \max } \forall i \in\left\{1, \ldots, n_{B}\right\}, \forall t \in\{1, \ldots, T\}
\end{aligned}
$$




$$
\begin{aligned}
& s_{c h}^{i, t}+s_{d c h}^{i, t} \leq 1 \forall i \in\left\{1, \ldots, n_{B}\right\}, \forall t \in\{1, \ldots, T\} \\
& \sum_{i=1}^{n_{B}} P_{c h}^{i, t}=\sum_{i=1}^{n_{m} C C H P} P_{m C C H P 2 B}^{i, t}+\sum_{i=1}^{n_{P V}} P_{P V 2 B}^{i, t}+P_{G 2 B}^{t} \forall t \in\{1, \ldots, T\} \\
& \sum_{\mathbf{i}=\mathbf{1}}^{\mathbf{n}_{\mathbf{B}}} \mathbf{P}_{\mathbf{d c h}}^{\mathbf{i}, \mathbf{t}}=\sum_{\mathbf{i}=\mathbf{1}}^{\mathbf{n}_{\mathbf{B}}} \mathbf{P}_{\mathbf{B 2 E L}}^{\mathbf{i}, \mathbf{t}} \forall \mathbf{t} \in\{\mathbf{1}, \ldots, \mathbf{T}\}
\end{aligned}
$$

\subsection{TL model}

The indoor temperature $\mathbf{T}_{\text {in }}^{\mathbf{i}, t}$ of the $\mathrm{i}$-th HAC in the building is expressed as (26) [22, 23]. $\mathrm{n}_{\text {HAC }}$ is the number of HAC appliances. $\mathbf{H}_{\mathrm{HAC}}^{\mathbf{i}}$ is the heat power consumed by the i-th HAC, $\mathbf{s}_{\mathrm{HAC}}^{\mathbf{i}, \mathbf{t}}$ on/off status of the $\mathrm{i}$-th HAC at time slot $\mathrm{t}$. $\mathbf{T}_{\mathbf{o u t}}^{\mathbf{t}}$ is the outdoor temperature, $\mathbf{c}_{\mathbf{a i r}}$ is the specific heat of air, $\mathrm{R}$ is the thermal resistance of the house wall. The hot water temperature $\mathbf{T}_{\mathbf{S W H}}^{\mathrm{j}, \mathbf{t}}$ of the $\mathrm{j}$-th storage water heater in the building is given as (27). $\mathrm{nSWH}$ is the number of SWH appliances. $\mathbf{H}_{\mathbf{S W H}}^{\mathbf{j}}$ is the heat power consumed by the $\mathrm{j}$-th $\mathrm{SWH}, \mathbf{s}_{\mathbf{S W H}}^{\mathrm{i}, \mathrm{t}}$ is the on/off status of the $\mathrm{j}$-th SWH at time slot $\mathrm{t} . \mathbf{T}_{\text {cold }}$ is the cold water temperature, $\mathbf{V}_{\text {cold }}^{\mathrm{j}, \mathrm{t}}$ is the volume of the cold water, $\mathrm{V}$ is the volume of the storage water heater, $\mathbf{C}_{\text {water }}$ is the specific heat of water. The constraints (28) and (29) ensure that the indoor temperature $\mathbf{T}_{\mathbf{i n}}^{\mathbf{i , t}}$ and $\mathbf{T}_{\mathbf{S W H}}^{\mathbf{j}, \mathbf{t}}$ are bounded between acceptable comfort ranges. $\mathbf{T}_{\mathbf{H A C}}^{\mathbf{i}, \text { min }}, \mathbf{T}_{\mathbf{S W H}}^{\mathbf{j}, \text { min }}$ and $\mathbf{T}_{\mathbf{H A C}}^{\mathbf{i}, \mathbf{m a x}}, \mathbf{T}_{\mathbf{S W H}}^{\mathbf{j}, \text { max }}$ are the minimal and maximal acceptable temperatures respectively, of the i-th $\mathrm{HAC}$ and hot water into the j-th SWH.

$$
\begin{aligned}
& T_{i n}^{i, t+1}=T_{i n}^{i, t} \cdot e^{\frac{-t}{R C_{a i r}}}+\left(R \cdot H_{H A C}^{i} \cdot s_{H A C}^{i, t}+T_{o u t}^{t}\right) \cdot\left(1-e^{\frac{-t}{R C_{a i r}}}\right) \forall i \in\left\{1, \ldots, n_{H A C}\right\}, \forall t \in\{1, \ldots, T\} \\
& T_{S W H}^{j, t+1}=\frac{V_{\text {cold }}^{j, t} \cdot\left(T_{\text {cold }}-T_{S W H}^{j, t}\right)+V \cdot T_{S W H}^{j, t}}{V}+\frac{H_{S W H}^{j} \cdot s_{S W H}^{j, t}}{V \cdot C_{\text {water }}} \forall j \in\left\{1, \ldots, n_{S W H}\right\}, \forall t \in\{1, \ldots, T\} \\
& T_{H A C}^{i, m i n} \leq T_{\text {in }}^{i, t} \leq T_{H A C}^{i, m a x} \forall i \in\left\{1, \ldots, n_{H A C}\right\}, \forall t \in\{1, \ldots, T\} \\
& T_{S W H}^{j, \min } \leq T_{S W H}^{j, t} \leq T_{S W H}^{j, \max } \forall j \in\left\{1, \ldots, n_{S W H}\right\}, \forall t \in\{1, \ldots, T\}
\end{aligned}
$$

\subsection{TSL model}

For each e $\in$ TSL, we define the load profile $\mathbf{q}_{\mathbf{e}}=\left\{\mathbf{q}_{\mathbf{e}}^{\mathbf{1}}, \mathbf{q}_{\mathbf{e}}^{\mathbf{2}}, \ldots, \mathbf{q}_{\mathbf{e}}^{\mathbf{D}^{\mathbf{e}}}\right\}$ where $\mathbf{q}_{\mathbf{e}}^{\mathbf{t}}$ is the required amount of electricity of e at time slot t. We denote $\mathbf{M S T} \mathbf{T}^{\mathbf{e}}, \mathbf{E} \mathbf{T}^{\mathbf{e}}$ and $\mathbf{D}^{\mathbf{e}}$ respectively the minimum starting time, the maximum ending time and the processing time of e-th TSL. $\mathrm{n}_{\mathrm{TSL}}$ is the number of TSL in the building. The electrical power $\mathbf{P}_{\mathbf{e}}^{\mathbf{t}}$ consumed by e-th TSL is formulated as (30) $\mathbf{x}_{\mathbf{e}}^{\mathbf{t}}$ is a binary variable which represents the working status of e in time slot $t . \mathbf{x}_{\mathbf{e}}^{\mathbf{t}}=\mathbf{1}$ if e starts at time $\mathrm{t}$ and 0 otherwise. The time window $\lambda \mathrm{e}$ of each e is defined as (31) [24]. The constraint (32) guarantees that each e-th TSL starts once within the time window $\lambda \mathrm{e}$.

$$
\begin{aligned}
& P_{e}^{t}=\sum_{d \in D^{e}, d \leq t} x_{e}^{t-d+1} \cdot q_{e}^{d} \forall e \in\left\{1, \ldots, n_{T S L}\right\} \forall t \in\{1, \ldots, T\} \\
& \lambda_{e}=\left[M S T^{e}, \ldots, E T^{e}-D^{e}\right] \forall e \in\left\{1, \ldots, n_{T S L}\right\} \\
& \left\{\begin{array}{c}
\sum_{t \in \lambda_{e}} \boldsymbol{x}_{\boldsymbol{e}}^{t}=\mathbf{1} \quad \forall \boldsymbol{e} \in\left\{\mathbf{1}, \ldots, \boldsymbol{n}_{T S L}\right\} \\
\boldsymbol{x}_{\boldsymbol{e}}^{t}=\mathbf{0} \quad \forall \boldsymbol{e} \in\left\{\mathbf{1}, \ldots, \boldsymbol{n}_{T S L}\right\}, \boldsymbol{t} \in \boldsymbol{T} \backslash \lambda_{\boldsymbol{e}}
\end{array}\right.
\end{aligned}
$$

\subsection{Other constraints}

The constraint (33) ensures that the total PVs power output should be equal to the power transmitted from all PVs to the electrical loads, battery and grid. The constraint (34) ensures that total mCCHP electrical power output should be equal to the power transmitted from all mCCHPs to the electrical loads, battery 
and grid. The electrical power balance is formulated in (35). $\mathbf{P}_{\mathbf{N C L}}^{\mathbf{t}}$ and $\mathbf{P}_{\mathbf{E C}}^{\mathbf{t}}$ are respectively the electrical demand of the non-controllable loads, and the electric chiller at time slot $t$. For cold weather conditions, the heat power balance is formulated in (36). For hot weather conditions, the cooling demand balance is formulated in (37) and (38) [25]. $\mathbf{C O P}^{\mathbf{A C}}$ and $\mathbf{H}_{\mathbf{A C}}^{\mathbf{i}, \mathbf{t}}$ are respectively the coefficient of performance and the heat power of the absorption chiller, and $\mathbf{C O P}^{\mathbf{E C}}$ is the coefficient of performance of the electric chiller.

$$
\begin{aligned}
& \sum_{i=1}^{n_{p v}} P_{P V}^{i, t}=\sum_{i=1}^{n_{p v}} P_{P V 2 E L}^{i, t}+\sum_{i=1}^{n_{p v}} P_{P V 2 B}^{i, t}+\sum_{i=1}^{n_{p v}} P_{P V 2 G}^{i, t} \forall t \in\{1, \ldots, T\} \\
& \sum_{i=1}^{n_{m C C H P}} P_{m C C H P}^{i, t}=\sum_{i=1}^{n_{m C C H P}} P_{m C C H P 2 E L}^{i, t}+\sum_{i=1}^{n_{m C C H P}} P_{m C C H P 2 B}^{i, t}+\sum_{i=1}^{n_{m C C H P}} P_{m C C H P 2 G}^{i, t} \\
& P_{N C L}^{t}+\sum_{i=1}^{n_{T S L}} P_{i}^{t}+P_{E C}^{t}=\sum_{i=1}^{n_{P V}} P_{P V 2 E L}^{i, t}+\sum_{i=1}^{n_{m C C H P}} P_{m C C H P 2 E L}^{i, t}+\sum_{i=1}^{n_{B}} P_{B 2 E L}^{i, t}+P_{G 2 E L}^{t} \\
& \sum_{i=1}^{n_{m C C H P}} H_{m C C H P}^{i, t}+\sum_{i=1}^{n_{T E S}} H_{i n}^{i, t} \cdot \eta_{i n}-\sum_{i=1}^{n_{T E S}} \frac{H_{d r}^{i, t}}{\eta_{d r}}=\sum_{i=1}^{n_{S W H}} s_{S W H}^{i, t} H_{S W H}^{i}+\sum_{i=1}^{n_{H A C}} s_{H A C}^{i, t} H_{H A C}^{i} \\
& \sum_{i=1}^{n_{m C C H P}} H_{m C C H P}^{i, t}+\sum_{i=1}^{n_{T E S}} H_{i n}^{i, t} \cdot \eta_{i n}-\sum_{i=1}^{n_{T E S}} \frac{H_{d r}^{i, t}}{\eta_{d r}}=\sum_{i=1}^{n_{S W H}} s_{S W H}^{i, t} H_{S W H}^{i}+\sum_{i=1}^{n_{m C C H P}} H_{A C}^{i, t}
\end{aligned}
$$

\subsection{Objectives}

\subsubsection{Total energy cost}

The total energy cost $F_{\text {cost }}$ over the scheduling horizon (cents/day) is formulated as (39) and composed of three parts; the electrical power cost imported from the grid penalized by a penalty factor $\delta$ if the violation of the maximum contractual peak power L between the utility and the smart building occurs, revenue from selling the surplus electrical power produced by the local energy sources to the grid as well as natural gas consumption cost of the $m C C H P . \pi_{\text {buy }}^{\mathrm{t}}, \pi^{\text {gas }}, \pi_{\text {sell,mCCHP }}$ and $\pi_{\text {sell,PV }}$ denote the utility electricity tariff for each time slot $t$, gas price, and $\mathrm{mCCHP}$ and PV feed-in tariffs respectively.

$$
\begin{aligned}
\min F_{\text {cost }} & =\sum_{t=1}^{T}\left(P_{G 2 E L}^{t}+P_{G 2 B}^{t}\right) \cdot \pi_{\text {buy }}^{t} \cdot 1_{\left(P_{G 2 E L}^{t}+P_{G 2 B}^{t}\right) \leq L}+\sum_{t=1}^{T}\left(P_{G 2 E L}^{t}+P_{G 2 B}^{t}\right) \cdot \delta \cdot \pi_{\text {buy }}^{t} \cdot 1_{\left(P_{G 2 E L}^{t}+P_{G 2 B}^{t}\right)>L} \\
& -\sum_{i=1}^{n_{P V}} \sum_{t=1}^{T} P_{P V 2 G}^{i, t} \cdot \pi_{\text {sell,PV }}-\sum_{i=1}^{n_{m C C H P}} \sum_{t=1}^{T} P_{m C C H P 2 G}^{i, t} \cdot \pi_{\text {sell,mCCHP }}+\sum_{i=1}^{n_{m C C H P}} \sum_{t=1}^{T} F_{m C C H P}^{i, t} \cdot \pi^{g a s}
\end{aligned}
$$

\subsubsection{Total discomfort}

The total discomfort objective $F_{\text {discomfort }}(\%)$ measures the quality of service of B-DSM, and it is divided into three parts; discomfort due to delay or advance operation of $T S L$, and discomforts caused by the deviation from desired indoor and storage water heater temperatures. $F_{\text {discomfort }}$ is modeled as expressed as (40). diss ${ }^{i, T S L}$ is formulated as (41). $S T^{e}$ and $P R E^{e}$ are respectively the current and preferred starting times of each e $\in T S L$. $\mathbf{T}_{\mathbf{i n}}^{\text {des }}$ and $\mathbf{T}_{\mathbf{h o t}}^{\text {des }}$ are the desired indoor and hot water storage temperatures respectively. 


$$
\begin{aligned}
& \min F_{\text {discomfort }}=\frac{1}{3}\left[100 \frac{1}{n_{T S L}} \sum_{e=1}^{n_{T S L}} d i s s^{e, T S L}+100 \frac{1}{n_{S W H}} \times \sum_{i=1}^{n_{S W H}} \frac{1}{T} \frac{\left|T_{\text {hot }}^{t}-T_{\text {hot }}^{\text {des }}\right|}{\max \left(T_{\text {hot }}^{\text {des }}-T_{S W H}^{\text {min }}, T_{S W H}^{\text {max }}-T_{\text {hot }}^{\text {des }}\right)}\right. \\
& \left.+100 \frac{1}{n_{H A C}} \sum_{i=1}^{n_{H A C}} \frac{1}{T} \frac{\left|T_{H A C}^{t}-T_{\text {in }}^{\text {des }}\right|}{\max \left(T_{i n}^{\text {des }}-T_{H A C}^{\min }, T_{H A C}^{\max }-T_{i n}^{\text {des }}\right)}\right] \\
& \operatorname{diss}^{e, T S L}=\left\{\begin{array}{c}
\frac{P R E^{e}-S T^{e}}{P R E^{e}-M S T^{e}} \text { if } M S T^{e} \leq S T^{e} \leq P R E^{e} \\
\frac{S T^{e}-P R E^{e}}{E T^{e}-D^{e}-P R E^{e}} \text { if } \mathrm{PRE}^{e} \leq S T^{e} \leq E T^{e}-D^{e}
\end{array}\right.
\end{aligned}
$$

\section{PROPOSED ALGORITHM}

\subsection{MOOP problem}

Many problems involve multiple conflicting objectives being optimized simultaneously. MOOP with such contradictory objectives lead to a set of optimal solutions instead of a single optimal solution. These optimal sets are known as Pareto-optimal solutions [16]. A MOOP problem can be defined as follows:

$$
(M O O P)=\left\{\begin{array}{c}
\left(f_{1}(x), f_{2}(x), \ldots, f_{q}(x)\right) \\
\text { s.t. } x \in X
\end{array}\right.
$$

$f(x)=\left(f_{1}(x), f_{2}(x), \ldots, f_{q}(x)\right)$ is the vector of $q$ objective functions $f_{i}$, and $q$ is the number of objectives $(q \leq 2)$. $x=\left(x_{1}, x_{2}, \ldots, x_{m}\right)$ represents the decision vector, where $x_{i}$ is a variable of the problem. $m$ is the number of decision variables. The set $\mathbb{R}$ which contains $X$ is called criteria or decision space. $Z=f(X)$ is the projection of the space $X$ onto the space of the objectives.

An objective vector $\mathrm{x}=\left(\mathrm{x}_{1}, \ldots, \mathrm{x}_{\mathrm{m}}\right) \in \mathrm{X}$ dominates another objective vector $\mathrm{x}^{\prime}=\left(\mathrm{x}^{\prime}{ }_{1}, \ldots, \mathrm{x}_{\mathrm{m}}^{\prime}\right) \in \mathrm{X}$ (denoted by $x \preccurlyeq x^{\prime}$ ) if and only if $x$ is partially less than $x^{\prime}$, i.e; $\forall i \in\{(1, \ldots, m)\}, x_{i} \leq x_{i}^{\prime} \wedge \exists i \in\{(1, \ldots, m)\}$, $\mathrm{x}_{\mathrm{i}}<\mathrm{x}_{\mathrm{i}}^{\prime}$. A solution $\mathrm{x} \in \mathrm{X}$ is said to be Pareto optimal if and only if there is no $\mathrm{x}^{\prime} \in \mathrm{X}$ such that $f\left(x^{\prime}\right)$ dominates $f(x)$. For a given MOOP, $f(x)$, the Pareto optimal set $P S$, is defined as:

$$
P S=\left\{x \in X / \nexists x^{\prime} \in X, f\left(x^{\prime}\right) \leq f(x)\right\}
$$

For a given MOOP, $f(x)$, and Pareto optimal set $P S$, the Pareto front $P F$ is defined as:

$$
P F=\left\{f(x) \in \mathbb{R}^{m} / x \in P S\right\}
$$

\subsection{H-NSGAII}

As explained earlier, H-NSGAII is a hybrid algorithm between NSGAII and CPLEX solver. By means of this hybridization, our matheuristic is capable to obtain a set of Pareto solutions in a reasonable time since CPLEX tackles only continuous variables, and we do not need to develop a specific heuristic for the feasible solution representation when applying any metaheuristic such as NSGAII. The implementation of our matheuristic H-NSGAII is described by the flowchart given in Figure 2.

\subsubsection{Step 1: Read input data}

In the first step, we gather input data related to our B-DSM, including the energy price market from the utility, the forcasted power generation of PV, outside temperature, technical specifications of each component in the building. We set the maximum number of generations, the number of population size.

\subsubsection{Step 2: Generate partial chromosomes}

In step 2, the adopted partial chromosome encoding scheme is based on a mixed binary and integervalued strings, each integer-valued gene encodes the starting time of each time shiftable load e $\in$ TSL, and randomly generated within $\lambda_{\mathrm{e}}$, whereas the binary genes encode the on/off status of thermal loads, the on/off status of mCCHP, TES heat power injected and drawn status, and batteries charging and discharging status for each time slot t. The length of each partial chromosome is: $\mathrm{n}_{\mathrm{TSL}}+\left(\mathrm{n}_{\mathrm{HAC}}+\mathrm{n}_{\mathrm{SWH}}+\mathrm{n}_{\mathrm{mCCHP}}+2 \times \mathrm{n}_{\mathrm{TES}}+2\right.$ $\left.\times \mathrm{n}_{\mathrm{B}}\right) \times \mathrm{T}$. 


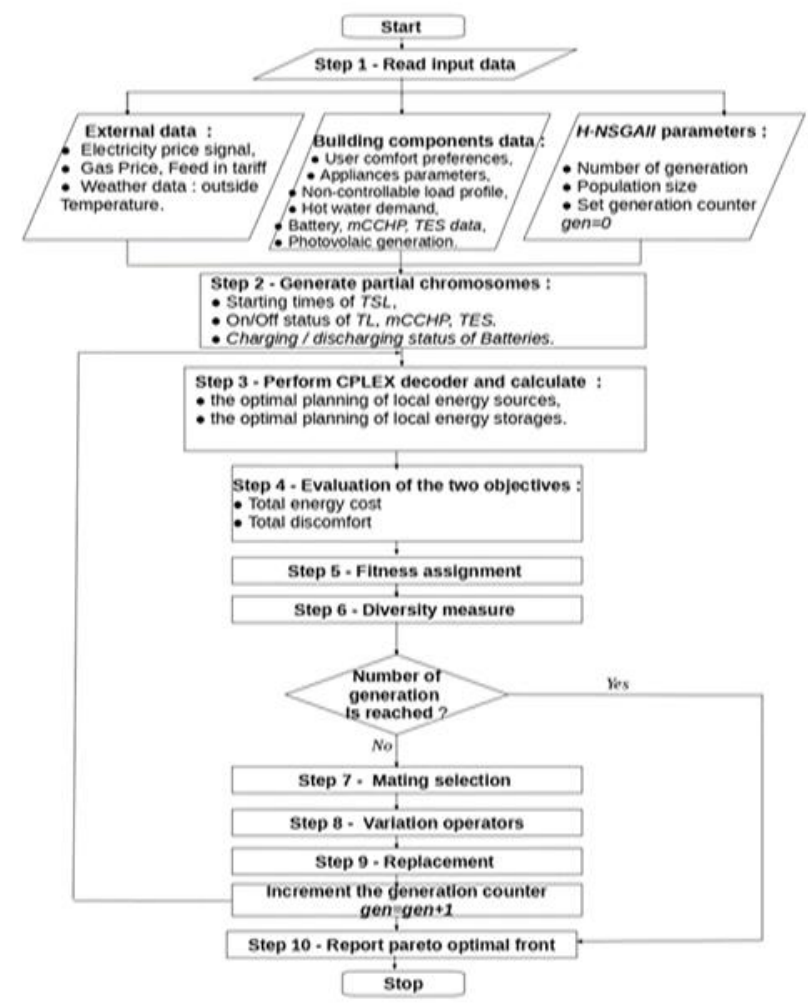

Figure 2. Proposed H-NSGAII

\subsubsection{Step 3: Perform CPLEX decoder}

In decoding step 3, the completeness of partial solution is carried out by solving a sub-problem to optimality using CPLEX solver. This sub-problem involves getting an optimal management of the local energy sources and storages. More specifically, the CPLEX decoder considers the discrete decision variables as input data provided from the previous step, and calculates to optimally the continuous variables.

\subsubsection{Step 4: Evaluate the two objective functions}

We evaluate the partial solutions of Popgen whose objective values are the total energy cost $F_{\text {cost }}$ and

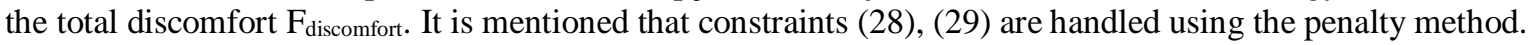

\subsubsection{Steps 5: Fitness assignment}

At each iteration gen, partial solutions are ranked based on the non-dominance concept [16].

\subsubsection{Step 6: Diversity measure}

Within a given non-dominance rank, partial solutions are sorted based on the crowding distance value [16]. Partial solutions with highest crowding distance are preferred because they introduce more diversity into the population.

\subsubsection{Step 7: Mating selection}

We use a binary tournament selection. Two partial solutions are randomly picked from the population. We select the best partial chromosome based on the non-dominance ranking. If their non-dominance rankings are equal, we select the partial solution that provides the largest value of the crowding distance.

\subsubsection{Step 8: Variation operators}

Variations operators are stochastic operators that modify partial solutions to progress in the feasible search space. In our B-DSM, a uniform crossover is adopted as depicted in Figure 3. Two offsprings are generated by swapping two randomly chosen starting times, HAC SWH, mCCHP, TES, batteries binary genes of two parents with a fixed probability prob ${ }_{\text {cross }}$. The mutation is carried out by changing a randomly chosen starting time, HAC, SWH, mCCHP, TES and batteries binary genes according to the mutation rate prob $_{\text {mut }}$ as indicated in Figure 4. 


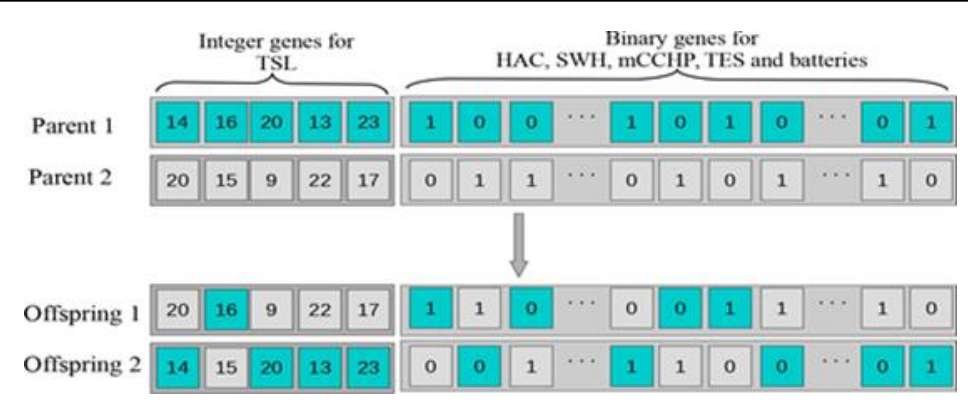

Figure 1. Crossover

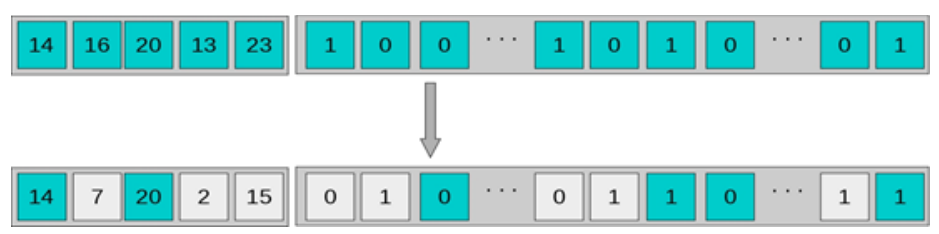

Figure 2. Mutation

\subsubsection{Step 9: Replacement}

An elitist replacement is carried out as follows: A population of offspring partial solutions $\mathrm{O}_{\text {gen }}$ is created with size Size. These partial chormosomes are decoded and evaluated. Then, a population $\mathrm{R}_{\text {gen }}$ with size $2 \times$ Size is obtained by combining $\mathrm{P}_{\text {gen }}$ and $\mathrm{O}_{\text {gen }}$. The partial solutions in $\mathrm{R}_{\text {gen }}$ are classified according to the non-dominance order and crowding distance values. The better half of the two populations' union is forming the new population.

\subsubsection{Step 10: Report Pareto optimal front} is recorded.

We iterate until the maximum number of generations is achieved. Finally, the Pareto optimal front

\section{SIMULATION RESULTS AND DISCUSSIONS}

\subsection{Data and parameters}

In this paper, a time horizon of 24 hours is considered and divided into 24 time slots of one hour each. For both hot and cold weather conditions, the time of use tariff as listed in Table 1. Day-ahead ToU is adopted. The natural gas tariff is 58.30 cents per $\mathrm{m} 3$. The feed-in tariffs are 12.00, 13.00 cents per $\mathrm{kWh}$ for hot and cold weather conditions respectively. We consider one mCCHP, one TES, one battery, one PV panel with a maximum power capacity of $6 \mathrm{kWh}$, three HAC, three SWH, and $18 \mathrm{TSL}$. The penalty factor $\delta$ is set to 3 . The contractual electrical power $\mathrm{L}$ is set to $4 \mathrm{~kW}$. The $\mathbf{T}_{\mathbf{u p}}^{\mathbf{i}, \mathbf{m a x}}, \mathbf{T}_{\mathbf{u p}}^{\mathbf{i}, \mathbf{m i n}}, \mathbf{T}_{\mathbf{d n}}^{\mathbf{i} \mathbf{m} \boldsymbol{i n}}$, COPAC and COPEC are set to $8,3,3,0.7,3$ respectively. The initial and final SOC are set to 0.5 . It is mentioned that all data for both cold and hot weather conditions used in this paper are available on-line at the mendeley repository [26.

Depending on these data and the components involved in the scheduling, experiments were grouped into 6 cases, three scenarios for hot weather conditions (SH1, SH2, SH3), and three scenarios for cold weather conditions (SC1, SC2, SC3) are taken into account. In SH1/SC1, PVs and batteries are not considered. In SH2/SC2, PVs are considered without batteries. In SH3/SC3, both PVs and batteries are taken into account.

Table 1. Day-ahead ToU

\begin{tabular}{|c|c|c|c|}
\hline & & $\begin{array}{l}\text { ToU for cold weather conditions } \\
\text { (Cents/kWh) }\end{array}$ & $\begin{array}{l}\text { ToU for hot weather conditions } \\
\text { (Cents/kWh) }\end{array}$ \\
\hline [8 A.M,14 P.M] and [20 P.M, 22 P.M] & Mid-peak & 27.00 & 28.00 \\
\hline [14 P.M, 20 P.M] & On-peak & 36.00 & 48.00 \\
\hline [22 P.M, 8 A.M] & Off-peak & 13.00 & 12.00 \\
\hline
\end{tabular}




\subsection{Comparison with CPLEX solver}

To assess the performance of our hybrid matheuristic H-NSGAII and to find out how our algorithm can cope with the limitations of prohibitive calcul time of exact solvers, and non-triviality of feasible solution encoding with the high number of equality and inequality constraints of the B-DSM optimization model, we compare H-NSGAII with the GP method solved with CPLEX for all six scenarios. As explained earlier, the introduction of discrete variables in the B-DSM makes the model hard and the computation time would grow exponentially. It is entirely acceptable to find good feasible Pareto solutions under reasonable computational time, even if the Pareto solutions obtained are suboptimal.

In the GP, we try to minimize the distance of objective functions from ideal point. Ideal point is calculated by solving each objective function separately. The multi-objective problem is transformed to a mono-objective problem to form a single objective problem as follows [27]:

$$
\begin{gathered}
\min v \cdot \frac{F_{\text {cost }}-F_{\text {cost }}^{\min }}{F_{\text {cost }}^{\max }-F_{\text {cost }}^{\min }}+\eta \cdot \frac{F_{\text {discomfort }}-F_{\text {discomfort }}^{\min }}{F_{\text {discomfort }}^{\max }-F_{\text {discomfort }}^{\min }} \\
\text { s.t (1). }-(38)
\end{gathered}
$$

where $\mathrm{F}_{\text {cost }}^{\min }, \mathrm{F}_{\text {discomfort }}^{\min }, \mathrm{F}_{\text {cost }}^{\max }$ and $\mathrm{F}_{\text {discomfort }}^{\max }$ are the minimum and the maximum values of $\mathrm{F}_{\text {cost }}$ and $\mathrm{F}_{\text {discomfort. }}$. $v$ and $\eta$ are the weights with $v+\eta=1$.

To obtain Pareto front in GP method, B-DSM is run multiple times and with different sets of weights. Each run gets one solution. The non-dominated solutions are extracted from these obtained solutions. The CPLEX time limit for each combination of weights is set to 15 seconds, the CPLEX number of runs is 100 .

The parameters of the proposed matheuristic H-NSGAII are as follows: The population size is 100 and the maximum generation number is 300 . The mutation probability is 0.5 and the crossover probability is 0.6 .

To validate and compare the proposed algorithms, we use four performance indicators:

- The computation time in seconds;

- The number of Pareto solutions found by each algorithm (NPS). Generally, the greater the NPS value is, the better the algorithm is;

- The hypervolume-difference $\left(\mathrm{I}_{\mathrm{H}}^{-}\right)$. It measures the quality of a Pareto set both in terms of convergence and diversity [28] .

- The convergence indicator $\epsilon$-indicator $\left(\mathrm{I}_{\epsilon}^{+}\right)[29,30]$.

Ten independent runs for each scenario are carried out using H-NSGAII. The reference Pareto front is generated by combining all found solutions of both algorithms and the Pareto solutions of this set form the reference front underlying the calculation of hypervolume difference and epsilon-indicator indicators. GP method is coded with the mathematical programming language (AMPL) and solved with CPLEX solver, and H-NSGAII algorithm is coded with ParadisEO version 2.0.1 [31], a C++ white-box object-oriented framework and Concert Technology with IBM ILOG CPLEX Optimization Studio Version 12.7.0. Both algorithms are run on a Linux 16.04 (64 bit) Intel@ Core i7-6500U CPU @ 2.50GHz $\times 4$ computer with an $8.00 \mathrm{GiB}$ memory.

Table 2 compares the average values of these indicators performed for each scenario. Obviously, H-NSGAII solves instances in remarkably less time than CPLEX. CPLEX cannot find the optimal Pareto solutions within a reasonable time and for some scenarios it stopped at CPLEX timelimit which is set to 1500 seconds. According to NPS indicator, H-NSGAII obtains more solutions than CPLEX, which offers more options to the decision-maker. As we have shown in the results of the hypervolume difference $\mathrm{I}_{\mathrm{H}}^{-}$and epsilon $\mathrm{I}_{\epsilon}^{+}$metrics for all scenarios, H-NSGAII is better than the CPLEX both in convergence and in spreading of the solutions sets. The results yields lower values of $\mathrm{I}_{\mathrm{H}}^{-}$and $\mathrm{I}_{\epsilon}^{+}$indicators. Consequently, the proposed H-NSGAII algorithm in this study can be regarded as an effective tool for solving our MOOP and mixedinteger linear problem in a reasonable time.

The compromise solutions and the extreme points of each objective $\left(\mathrm{F}_{\text {cost }}, \mathrm{F}_{\text {discomfort }}\right)$ are compared for both algorithms in Table 3. It is mentionned that the compromise solutions are selected using TOPSIS method [32]. As we can see from this table, H-NSGAII outperforms GP in all scenarios. This is due to the crowding distance mechanism of H-NSGAII algorithm which preserves diversity of solutions and maintains the extreme points at each iteration. In the other hand, for both hot and cold weather scenarios, we can also observe that the scenarios SH3 and SC3 can achieve more cost savings than others scenarios. This is due the fact that the combination between all components and flexible loads motive our B-DSM framework to more efficiently utilize the energy sources and storages. 
Pareto fronts obtained for both algorithms on the SC3 and SH3 scenarios are shown in Figure 5 (a) and Figure 5 (b). The compromise and extreme solutions of H-NSGAII are marked in these figures. From these figures, it is clear that the applied H-NSGAII is able to generate Pareto front in a single simulation run. Each solution point on the Pareto front indicates a non-dominated B-DSM scheduling operation strategy, where the trade-off between the total energy cost, and the discomfort is achieved. We can see that the Pareto solutions for the GP are not evenly distributed. Solutions obtained by the proposed matheuristic are distributed more uniformly in comparison with the GP.

Table 2. Performance metrics comparison between H-NSGAII and GP

\begin{tabular}{|c|c|c|c|c|c|c|}
\hline & Instances & Algorithm & Time (s) & NPS & $I_{H}^{-}$ & $I_{\epsilon}^{+}$ \\
\hline \multirow{6}{*}{$\begin{array}{l}\text { Hot weather } \\
\text { conditions }\end{array}$} & \multirow[t]{2}{*}{ SH1 } & H-NSGAII & 283 & 178 & $3.54 \mathrm{E}-04$ & $7.78 \mathrm{E}-03$ \\
\hline & & CPLEX & 1500 & 28 & $4.27 \mathrm{E}-02$ & $6.77 \mathrm{E}-02$ \\
\hline & \multirow[t]{2}{*}{$\mathrm{SH} 2$} & H-NSGAII & 411.7 & 118 & $4.58 \mathrm{E}-04$ & $7.57 \mathrm{E}-03$ \\
\hline & & CPLEX & 1500 & 25 & $1.24 \mathrm{E}-02$ & $2.86 \mathrm{E}-02$ \\
\hline & \multirow[t]{2}{*}{ SH3 } & H-NSGAII & 420 & 184 & $1.23 \mathrm{E}-04$ & $2.56 \mathrm{E}-03$ \\
\hline & & CPLEX & 1500 & 24 & $4.29 \mathrm{E}-02$ & $6.91 \mathrm{E}-02$ \\
\hline \multirow{6}{*}{$\begin{array}{l}\text { Cold weather } \\
\text { conditions }\end{array}$} & \multirow[t]{2}{*}{ SC1 } & H-NSGAII & 46.39 & 171 & 7.13E-03 & $4.62 \mathrm{E}-02$ \\
\hline & & CPLEX & 1206.6 & 28 & $3.45 \mathrm{E}-02$ & $6.59 \mathrm{E}-02$ \\
\hline & \multirow[t]{2}{*}{$\mathrm{SC} 2$} & H-NSGAII & 94.13 & 183 & $4.90 \mathrm{E}-03$ & $3.07 \mathrm{E}-02$ \\
\hline & & CPLEX & 1264.8 & 21 & $1.16 \mathrm{E}-01$ & $1.30 \mathrm{E}-01$ \\
\hline & \multirow[t]{2}{*}{$\mathrm{SC} 3$} & H-NSGAII & 392.06 & 115 & $4.96 \mathrm{E}-04$ & $8.46 \mathrm{E}-03$ \\
\hline & & CPLEX & 1500 & 26 & $1.34 \mathrm{E}-01$ & $1.71 \mathrm{E}-01$ \\
\hline
\end{tabular}

Table 3. Extreme and compromise solutions for all scenarios for H-NSGAII and GP algorithms

\begin{tabular}{llllll}
\hline & Instances & Algorithm & Best energy cost solution & Best discomfort solution & Compromise solution \\
\hline $\begin{array}{l}\text { Hot weather } \\
\text { conditions }\end{array}$ & SH1 & H-NSGAII & $(1704.7635 .5819)$ & $(8787.45 .98074)$ & $(2394.2112 .3299)$ \\
& & CPLEX & $(1761.06034 .4232)$ & $(8698.297 .30403)$ & $(2416.8913 .0833)$ \\
& SH2 & H-NSGAII & $(1009.6745 .6252)$ & $(5916.87 .09835)$ & $(1678.7810 .0841)$ \\
& & CPLEX & $(1062.7541 .8978)$ & $(6516.2407 .13709)$ & $(1695.310 .3254)$ \\
& SH3 & H-NSGAII & $(1005.5347 .0681)$ & $(4952.247 .11)$ & $(1298.9110 .71)$ \\
Cold weather & SC1 & CPLEX & $(104231.13360)$ & $(4978.917 .25895)$ & $(1413.2510 .8291)$ \\
conditions & & H-NSGAII & $(2480.6835 .4239)$ & $(8169.497 .1336)$ & $(3669.6313 .1882)$ \\
& SC2 & CPLEX & $(2480.9737 .7693)$ & $(8182.077 .29231)$ & $(4001.2713 .444)$ \\
& & H-NSGAII & $(2359.6839 .4373)$ & $(7555.676 .53384)$ & $(3292.07013 .1822)$ \\
& SC3 & CPLEX & $(2384.4630 .7114)$ & $(7864.697 .3988)$ & $(3305.9714 .2801)$ \\
& & H-NSGAII & $(2251.4938 .1855)$ & $(6672.147 .2)$ & $(2696.311 .8098)$ \\
\end{tabular}

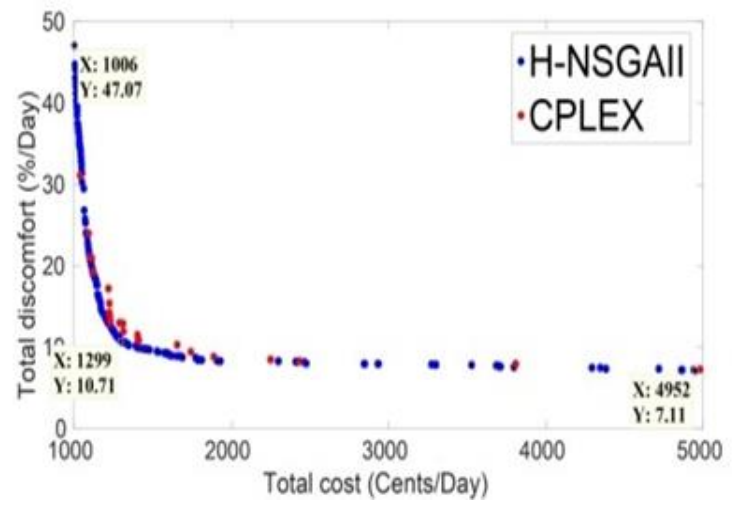

(a)

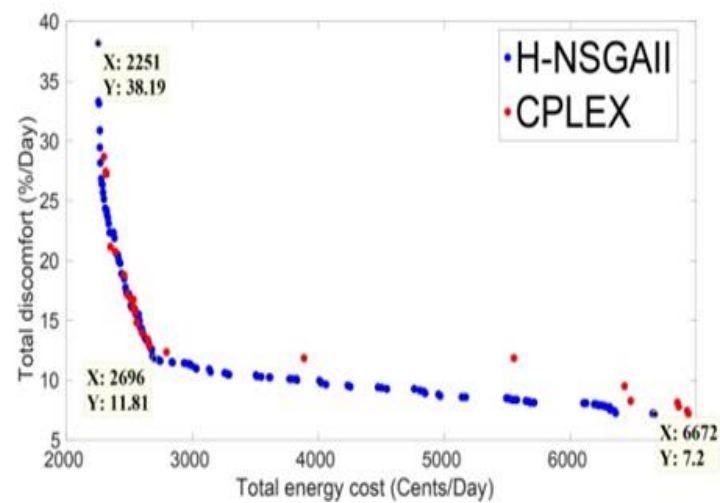

(b)

Figure 5. Pareto front, (a) SH3, (b) SC3

\subsection{Comparison between extreme and compromise solutions}

In this subsection, we study some particular solutions from the Pareto front of SH3 scenario, including the best cost solution, the best discomfort solution and the compromise solution in order to show the operation load scheduling behaviour of TL (HACs, SWHs) and TSL of these solutions. Figure 6 and Figure 7 shows the indoor and hot water temperature profiles for SH3. 


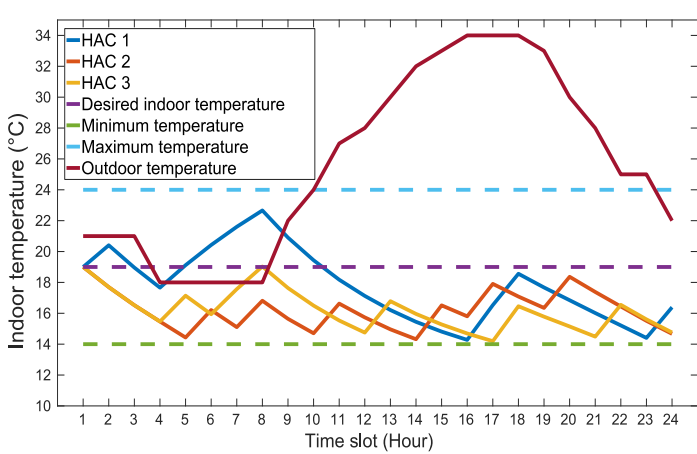

(a)

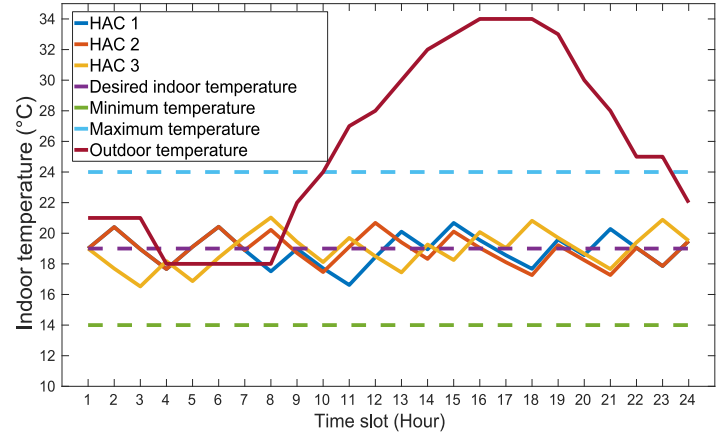

(b)

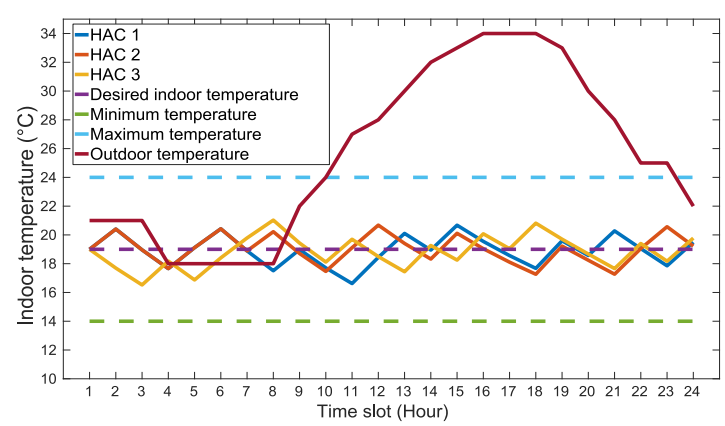

(c)

Figure 6. Indoor temperature for SH3 for the three selected Pareto solutions (a) Best cost solution, (b) Best discomfort solution, (c) Compromise solution

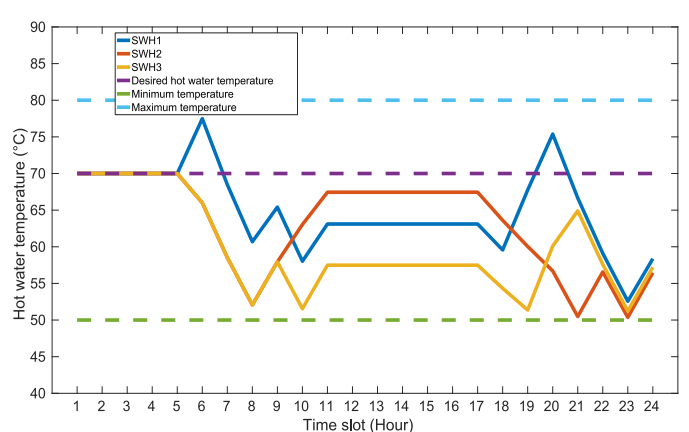

(a)

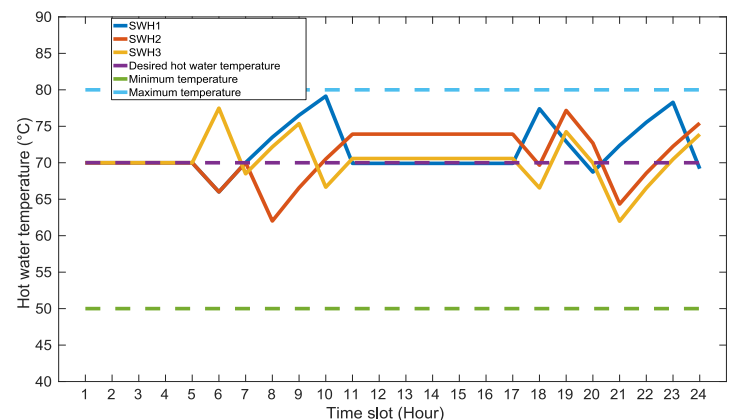

(b)

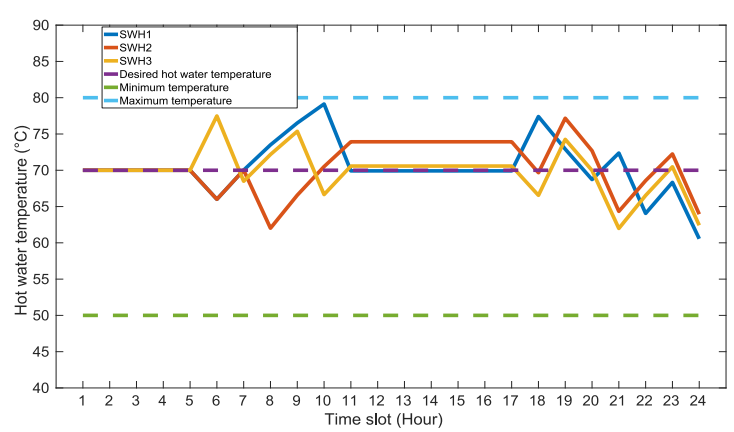

(c)

Figure 7. Hot water temperature for $\mathrm{SH} 3$ for the three selected Pareto solutions, (a) Best cost solution, (b) Best discomfort solution (c) Compromise solution 
Figure 6 and Figure 7 shows the indoor and hot water temperature profiles for SH3. In the same figures, the minimum, the maximum and the preferred temperatures are also plotted. As we can see, the indoor and hot water temperatures are within their prespecified ranges. For the three considered HAC and $\mathrm{SWH}$, the indoor and hot water temperatures are much closer to the user preferred temperatures in almost all time slots for the best discomfort and compromise solutions compared to the best cost solutions.

Figure 8 depicts the electrical power dispatch and state of different electrical loads for SH3 for the three selected Pareto solutions. For the best cost and compromise solutions, the electrical power bought from the grid (G2EL) is under the contractual power limit $L$ and the import of electricity is shifted to the low price periods, however for the best discomfort solution the contractual power limit is violated between time slot 14 and 17 which increases the energy cost.

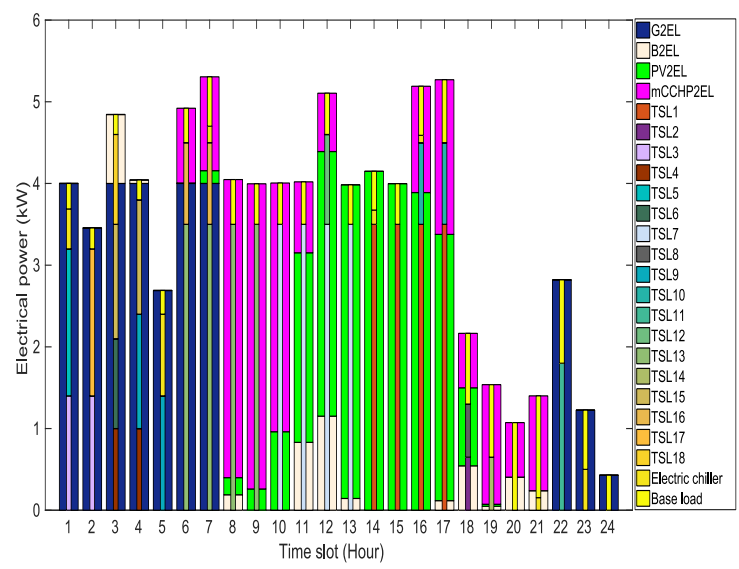

(a)

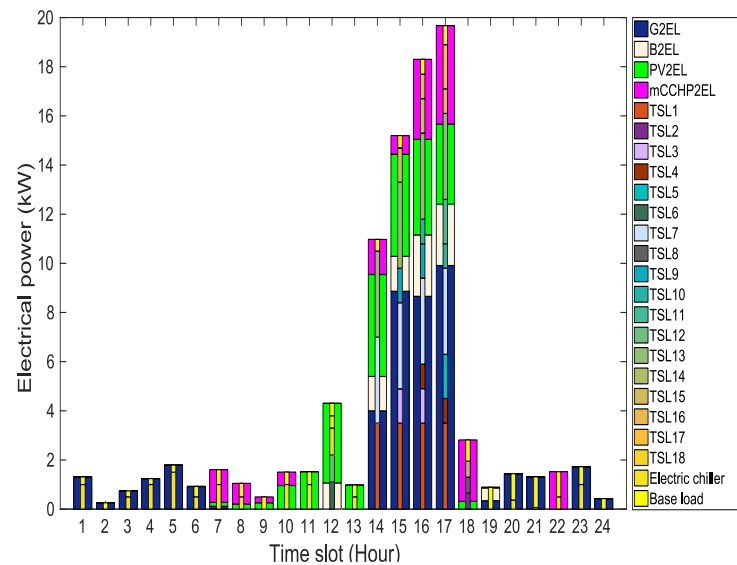

(b)

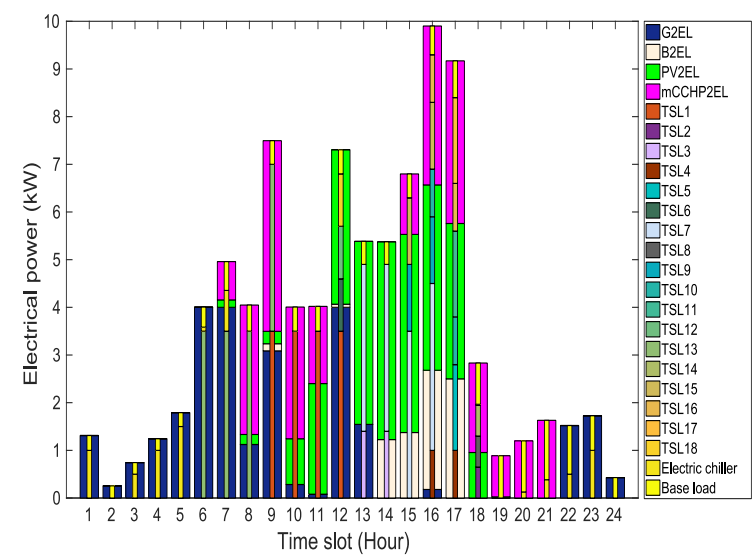

(c)

Figure 8. Electrical power dispatch for SH3 for the three selected Pareto solutions (a) Best cost solution, (b) Best discomfort solution, (c) Compromise solution

Figure 9 (a) shows the mCCHP heat production at each time slot for the compromise solution of SH3 scenario. As we can see, the operational constraints minimum/maximum Up-Time, and minimum Down-Time constraints are satisfied. The mCCHP reaches its maximum at time slot 9 and nearly the maximum at time slots 16 and 17. The thermal energy content and the heat power injected and drawn for the compromise solution of SH3 scenario is shown in Figure 9 (b). As can be seen, the TES profile fall between their maximum and minimum values. The initial and final TES content are set to $10 \mathrm{kWh}$. The battery SOC and power for the compromise solution of SH3 scenario are depicted in Figure 9 (c). The positive and negative values represent the battery charging and discharging, respectively. For this Pareto solution, the battery charges from the grid during low price periods and discharges during peak price to help satisfy loads and keep the electrical demand from the grid under the contractual power limit. The SOC of the domestic battery is within the specified range during the scheduling horizon. 


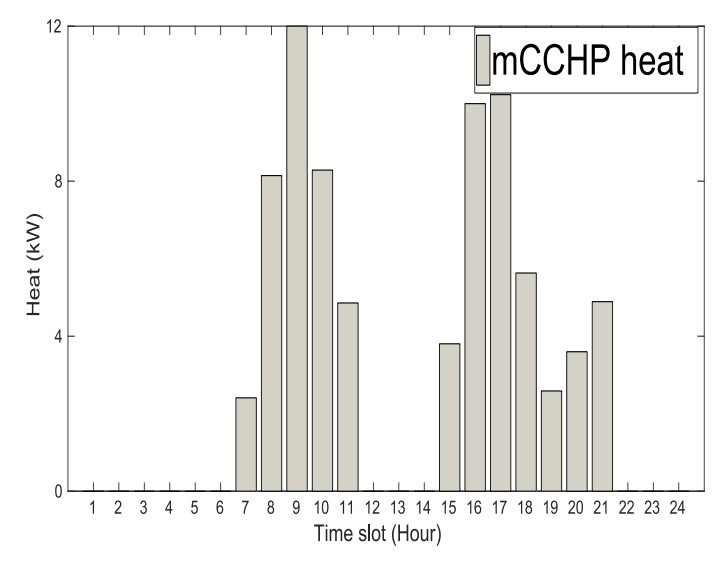

(a)

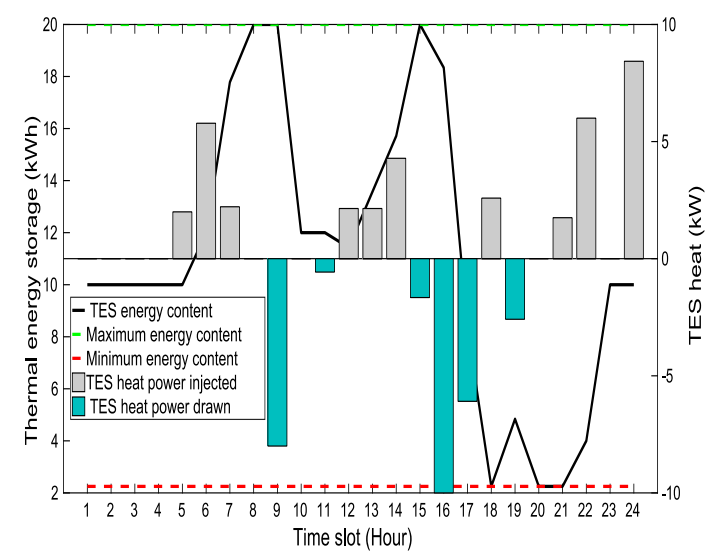

(b)

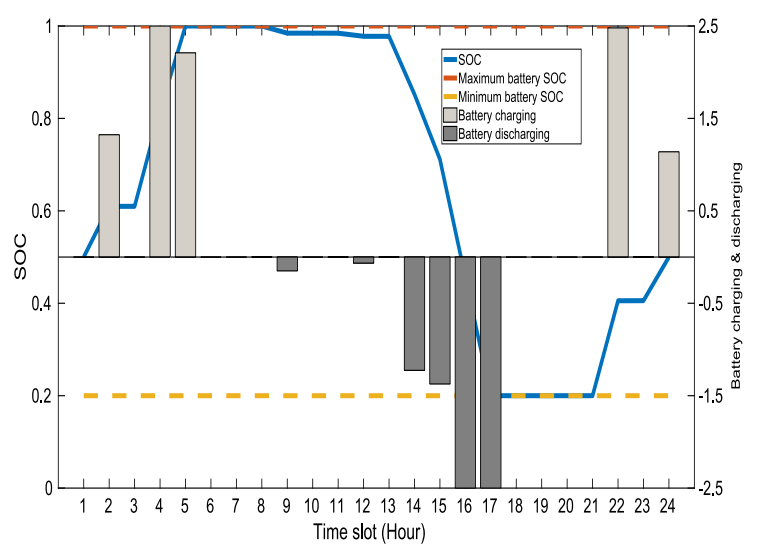

(c)

Figure 9. The mCCHP, TES, and battery operations for the compromise solution of SH3, (a) mCCHP heat power output, (b) TES energy content with the heat drawn and injected,

(c) Battery SOC with the charging and discharging power

\section{CONCLUSION}

In this paper, we have proposed a B-DSM optimization model to schedule electrical and thermal appliances with local energy sources (mCCHP, PV) and storages (TES, Battery). The B-DSM is formulated as a mixed-integer linear and multi-objective optimization problem, the bi-objective problem deals with reducing simultaneously of the total energy cost, and the total discomfort caused by allowing flexibility of loads. To deal with the restrictions of excessif computational time of CPLEX with the number of discrete variables considered in the model, and the nontriviality of feasible solutions encoding with the number of equality and inequality constraints when implementing a MOEA, we develop a matheuristic algorithm based on combining the NSGAII algorithm and a black box solver to tackle this problem. In this approach, discrete variables are encoded as partial solutions by the NSGAII and considered as input data for the CPLEX solver which optimally determines the continuous variables. This matheuristic is effective for our constrained MOOP when neither a black box solver nor a MOEA can find good feasible solutions in a reasonable time.

Several simulations are carried out for both cold and hot weather conditions and compared with the GP solved with CPLEX under six scenarios. The results demonstrate the effectiveness of our proposed framework and show that our matheuristic is able to obtain more Pareto solutions in a much shorter time that are far better than those obtained by CPLEX according to the hypervolume difference and epsilon indicator metrics. Future extensions to this work will be mainly aimed at investigating data uncertainties due to the penetration of volatile renewable energy sources, electricity and gas prices forecasting errors and unpredictable energy consumption. We will also formulate our B-DSM to encompass several buildings by taking into account energy trading between buildings through so-called collaborative micro-grids. 


\section{REFERENCES}

[1] C. Wilson, T. Hargreaves, and R. Hauxwell-Baldwin, "Smart homes and their users: a systematic analysis and key challenges," Pers. Ubiquitous Comput., vol. 19, no. 2, pp. 463-476, 2015.

[2] M. Alaa, A. Zaidan, B. Zaidan, M. Talal, and M. Kiah, "A review of smart home applications based on Internet of Things," J. Netw. Comput. Appl., vol. 97, pp. 48-65, 2017.

[3] J. S. Vardakas, N. Zorba, and C. V. Verikoukis, "survey on demand response programs in smart grids: Pricing methods and optimization algorithms," IEEE Commun. Surv. Tutor., vol. 17, no. 1, pp. 152-178, 2015.

[4] K. Ma, S. Hu, J. Yang, X. Xu, and X. Guan, "Appliances scheduling via cooperative multi-swarm PSO under dayahead prices and photovoltaic generation," Appl. Soft Comput., vol. 62, pp. 504-513, 2018.

[5] D. Setlhaolo, X. Xia, and J. Zhang, "Optimal scheduling of household appliances for demand response," Electr. Power Syst. Res., vol. 116, pp. 24-28, 2014.

[6] Yanyu Zhang Peng Zeng, Shuhui Li, Chuanzhi Zang and H. Li, "A Novel Multiobjective Optimization Algorithm for Home Energy Management System in Smart Grid," Math. Probl. Eng., vol. 2015, pp. 19, 2015.

[7] A. Anvari-Moghaddam, H. Monsef, and A. Rahimi-Kian, "Optimal Smart Home Energy Management Considering Energy Saving and a Comfortable Lifestyle," Smart Grid IEEE Trans. On, vol. 6, no. 1, pp. 324-332, Jan. 2015.

[8] A. Soares, C. H. Antunes, C. Oliveira, and Á. Gomes, "A multi-objective genetic approach to domestic load scheduling in an energy management system," Energy, vol. 77, pp. 144-152, 2014.

[9] M. R. Alam, M. St-Hilaire, and T. Kunz, "An optimal P2P energy trading model for smart homes in the smart grid," Energy Effic., May 2017.

[10] M. R. Sandgani and S. Sirouspour, "Energy Management in a Network of Grid-Connected Microgrids/Nanogrids Using Compromise Programming," IEEE Trans. Smart Grid, vol. 9, no. 3, pp. 2180-2191, May 2018.

[11] S. Amiri, M. Honarvar, and A. sadegheih, "Providing an integrated Model for Planning and Scheduling Energy Hubs and preventive maintenance," Energy, vol. 163, pp. 1093-1114, 2018.

[12] M. Ross, C. Abbey, F. Bouffard, and G. Jos, "Multiobjective Optimization Dispatch for Microgrids with a High Penetration of Renewable Generation," IEEE Trans. Sustain. Energy, vol. 6, no. 4, pp. 1306-1314, Oct. 2015.

[13] M. R. Sandgani and S. Sirouspour, "Coordinated Optimal Dispatch of Energy Storage in a Network of GridConnected Microgrids," IEEE Trans. Sustain. Energy, vol. 8, no. 3, pp. 1166-1176, 2017.

[14] Ş. Gür and T. Eren, "Scheduling and Planning in Service Systems with Goal Programming: Literature Review," Mathematics, vol. 6, no. 11, 2018.

[15] K. Deb and D. Kalyanmoy, Multi-Objective Optimization Using Evolutionary Algorithms. New York, NY, USA: John Wiley \& Sons, Inc., 2001.

[16] E.-G. Talbi, Metaheuristics: From Design to Implementation, Wiley Publishing, 2009.

[17] V. Maniezzo, et al., Matheuristics-Hybridizing Metaheuristics and Mathematical Programming, Springer, 2010.

[18] H. Yousefi, M. H. Ghodusinejad, and A. Kasaeian, "Multi-objective optimal component sizing of a hybrid ICE+ PV/T driven CCHP microgrid," Appl. Therm. Eng., vol. 122, pp. 126-138, 2017.

[19] F. Brahman, M. Honarmand, and S. Jadid, "Optimal electrical and thermal energy management of a residential energy hub, integrating demand response and energy storage system," Energy Build., vol. 90, pp. 65-75, 2015.

[20] J. Wang, et al., "Impact of wind power forecasting on unit commitment and dispatch," in Proc. 8th Int. Workshop Large-Scale Integration of Wind Power into Power Systems, pp. 1-8, 2009.

[21] P. Liu, Y. Fu, and A. Kargarian marvasti, "Multi-stage stochastic optimal operation of energy-efficient building with combined heat and power system," Electr. Power Compon. Syst., vol. 42, no. 3-4, pp. 327-338, 2014.

[22] M. Tasdighi, et al., "Residential Microgrid Scheduling Based on Smart Meters Data and Temperature Dependent Thermal Load Modeling," IEEE Trans. Smart Grid, vol. 5, no. 1, pp. 349-357, Jan. 2014.

[23] M. A. A. Pedrasa, T. D. Spooner, and I. F. MacGill, "Coordinated Scheduling of Residential Distributed Energy Resources to Optimize Smart Home Energy Services," IEEE Trans. Smart Grid, vol. 1, no. 2, pp. 134-143, 2010.

[24] J. Soares, M. Silva, T. Sousa, Z. Vale, and H. Morais, "Distributed energy resource short-term scheduling using Signaled Particle Swarm Optimization," Energy, vol. 42, no. 1, pp. 466-476, 2012.

[25] M. Shahnazari, et al., "Equipment capacity optimization of an educational building's CCHP system by genetic algorithm and sensitivity analysis," Energy Equip. Syst., vol. 5, no. 4, pp. 375-387, 2017.

[26] Z. Garroussi, Data for energy management in a smart home, Mendeley, 2019.

[27] H. Farrokhi-Asl, M. Rabbani, and B. Asgarian, "Solving a bi-objective location routing problem by a NSGA-II combined with clustering approach: application in waste collection problem," J. Ind. Eng. Int., vol. 13, 2016.

[28] E. Zitzler and L. Thiele, "Multiobjective evolutionary algorithms: a comparative case study and the strength Pareto approach," IEEE Trans. Evol. Comput., vol. 3, no. 4, pp. 257-271, Nov. 1999.

[29] E. Zitzler, L. Thiele, M. Laumanns, C. M. Fonseca, and V. G. Da Fonseca, "Performance assessment of multiobjective optimizers: An analysis and review," IEEE Trans. Evol. Comput., vol. 7, no. 2, pp. 117-132, 2003.

[30] C. M. Fonseca, J. Knowles, L. Thiele, and E. Zitzler, "A tutorial on the performance assessment of stochastic multiobjective optimizers," Tik Rep., vol. 216, 2006.

[31] A. Liefooghe, et al., "ParadisEO-MOEO: A framework for evolutionary multi-objective optimization," in International Conference on Evolutionary Multi-Criterion Optimization, pp. 386-400, 2007.

[32] M. A. Abido, "Multiobjective evolutionary algorithms for electric power dispatch problem," IEEE Trans. Evol. Comput., vol. 10, no. 3, pp. 315-329, 2006. 